\title{
Achalasia: A to Z
}

\author{
John G. Hunter ${ }^{1}$
}

Published online: 4 April 2015

(C) Société Internationale de Chirurgie 2015

For reasons that only partially escape me, many of the world's experts in the surgical care of achalasia hail from Italy. We have the good fortune to have many of them contributing to this symposium, from Allaix to Zaninotto. To assure the true international flavor of this symposium additional experts from Argentina, Brazil, Germany, and the US have been assembled to create an "all-star cast", under the direction of guest editor and guru, Marco Patti. The resulting ten piece symposium should serve as the guide for any surgeon managing achalasia today. The summary points of this symposium, if I might be so bold, are as follows:

(1) New technology has brought new understanding of the pathophysiology of achalasia. High resolution motility (HRM) and the Chicago classification system based on HRM has redefined achalasia into three distinct categories. Treatment approach and treatment outcome have been observed to differ across the three achalasia variants defined by the Chicago classification.

(2) Heller myotomy with partial fundoplication remains the "gold standard" treatment for achalasia. Alternative therapies, including balloon dilation, botulinum toxin injection, peroral, myotomy (POEM), and esophagectomy, have distinct and emerging roles in treating achalasia in certain circumstances (a) where the diagnosis of achalasia may be in doubt, (b) where Heller myotomy might be too risky (elderly),

John G. Hunter

hunterj@ohsu.edu

1 Department of Surgery, School of Medicine, Oregon Health and Science University, Portland, Oregon, USA

(3) The treatment of epiphrenic diverticulum is suitably "lumped", in this symposium with achalasia, as the primary treatment is Heller myotomy, extending to a level above the neck of the diverticulum. The primary objective is to relieve the outflow obstruction to the esophagus and to diminish the high pressures in the distal esophagus that caused the diverticulum to be blown through the wall of the esophagus in the first place. Diverticulectomy is the secondary objective, and in many circumstances diverticulectomy may not be necessary.

(4) The 'new kid on the block' is peroral endoscopic myotomy (POEM). This operation is very appealing as it offers the surgical precision of lower esophageal sphincter (LES) division under direct vision with an approach more minimally invasive than Heller myotomy. Longitudinal (5 year minimum) comparative studies of POEM versus Heller myotomy, or POEM vs balloon dilation are yet to be done. Not until these studies are complete will we know the true role of POEM in the management of achalasia. Until then, it is imperative that the surgeon offering a POEM or-for that matter-any new or innovative procedure follow the steps recommended by the Balliol College group or the American College of Surgeons to insure that patient choice and patient safety are optimized.

As you have surely discerned, these four summary points are brief, shallow, and incomplete. They should not serve as any sort of a "take away" from this symposium, but only as a teaser to welcome you to ten very interesting 
articles that need to be read their entirety in order to understand achalasia and its management in depth. The management of complex esophageal motility disorders is full of traps for the novice, so dig into these articles, and pay attention to the details, to the pearls of wisdom of the experts, gained from extensive experience in caring for patients with achalasia and its several cousins. 